Artículo científico

Volumen 32(2):538-555. Mayo-agosto, 2021

e-ISSN 2215-3608, doi:10.15517/am.v32i2.41265

https://revistas.ucr.ac.cr/index.php/agromeso/index

\title{
Adaptación de mezclas forrajeras a diferentes niveles de enmienda y riego en Nariño, Colombia ${ }^{1}$
}

\section{Adaptation of fodder mixtures at different levels of amendment and irrigation in Nariño, Colombia}

\author{
Paola Andrea Portillo-López $z^{2}$, Diego Hernán Meneses-Buitrago², Elizabeth Lagos-Burbano², \\ María E. Duter-Nisivoccia ${ }^{3}$ Edwin Castro-Rincón ${ }^{2}$
}

1 Recepción: 24 de abril, 2020. Aceptación: 22 de setiembre, 2020. Este trabajo formó parte del proyecto de tesis de Maestría del primer autor, llevado a cabo en el marco del proyecto denominado "Mejoramiento de la oferta forrajera, optimización de los sistema de alimentación y aseguramiento de la calidad e inocuidad de la leche en el trópico alto del departamento de Nariño", financiado por el Sistema General de Regalías y Gobernación de Nariño, ejecutado por el Centro de Investigación Obonuco de la Corporación Colombiana de Investigación Agropecuaria (AGROSAVIA), Pasto, Nariño.

2 Corporación Colombiana de Investigación Agropecuaria (AGROSAVIA), Centro de Investigación Obonuco, Pasto, Colombia. pportillo@agrosavia.co (https://orcid.org/0000-0003-1189-9173), dmeneses@agrosavia.co (https://orcid.org/0000-0003-3033-3079), elizalagosb@hotmail. com (https://orcid.org/0000-0002-5098-9908), ecastro@agrosavia.co (autor para la correspondencia, https://orcid.org/0000-0001-9841-8242).

3 Seed Force Ltd. Canterbury \& West Coast. Hornby, Christchurch, New Zealand. latinlincuru@ gmail.com (https://orcid.org/0000-0003-35143020).

\section{Resumen}

Introducción. La ganadería de leche especializada del trópico alto de Nariño se enfrenta a diferentes limitantes, como la variabilidad y estacionalidad de la oferta forrajera. Objetivo. Evaluar seis mezclas de forrajes perennes, anuales y leguminosas, tres niveles de enmienda y dos niveles de riego en el trópico alto de Nariño. Materiales y métodos. El estudio se desarrolló entre diciembre de 2017 y noviembre de 2018, en el centro de investigación Obonuco de la Corporación Colombiana de Investigación Agropecuaria. Se utilizó un diseño de bloques completos al azar, con un arreglo de parcelas subdivididas. Se midió: vigor, altura, cobertura, número de hojas, incidencia de plagas, incidencia y severidad de enfermedades, deficiencias nutricionales y materia seca (MS). Para el análisis se empleó el software R V.3.6.0. Resultados. La asociación (gramínea y leguminosa) generó un efecto directo sobre el rendimiento de MS, cobertura y número de hojas, la mejor mezcla fue la compuesta por Dactylis glomerata,Trifolium repens L. y Trifolium pratense L. Para la variable altura los mayores valores los presentó la mezcla Tetrablend 260. La mayoría de tratamientos se mantuvieron en escalas sin daño y daño leve para las variables incidencia de plagas, severidad de enfermedades y deficiencias nutricionales. Conclusiones. Las mezclas de gramíneas y leguminosas presentaron ventajas comparativas frente al monocultivo de gramíneas puras, en cuanto a rendimiento de MS y variables agronómicas. En general, todos los tratamientos se mantuvieron en umbrales de daño mínimo y leve para las variables fitosanitarias.

Palabras clave: gramíneas, leguminosas, materia seca, estacionalidad, plantas forrajeras. 


\begin{abstract}
Introduction. Specialized dairy farming in the high tropics of Nariño faces with different limitations, such as the variability and seasonality of the fodder supply. Objective. To evaluate six mixtures of perennial, annual, and leguminous fodder, three levels of amendment and two levels of irrigation in the high tropic of Nariño. Materials and methods. The study was developed between December 2017 and November 2018 at the Obonuco research center of the Colombian Agricultural Research Corporation. A complete randomized block design was used, with an arrangement of subdivided plots. It was measured: vigor, height, coverage, number of leaves, incidence of pests, incidence and severity of diseases, nutritional deficiencies, and dry matter (MS). Software R V.3.6.0 was used for the analysis. Results. The association (gramineous and leguminous) generated a direct effect on the yield of MS, cover, and number of leaves, being the best mixture the one composed by Dactylis glomerata, Trifolium repens L., and Trifolium pratense L. For the variable height, the highest values were presented in the Tetrablend 260 mixture. Most of the treatments were kept on scales without damage and slight damage for the variables incidence of pests, severity of diseases and nutritional deficiencies. Conclusion. The mixtures of grasses and legumes showed comparative advantages over pure grass monoculture in terms of MS yield and agronomic variables. In general, all the treatments were kept in minimum and light damage thresholds for the phytosanitary variables.
\end{abstract}

Keywords: grasses, legumes, dry matter, seasonality, feed crops.

\title{
Introducción
}

Los forrajes constituyen la alternativa de alimentación predominante en los diferentes sistemas de producción bovina, son la fuente más económica para satisfacer el consumo voluntario de los rumiantes y se requieren para garantizar su adecuada fisiología ruminal (Reynolds, 2000).

En el departamento de Nariño, la base forrajera para los sistemas ganaderos está constituida principalmente por el pasto kikuyo, gramínea predominante en un $80 \%$ en los sistemas de lechería especializada, el cual ha demostrado muy buena adaptación. No obstante, este pasto presenta baja productividad, debido, entre otros factores, a las deficientes prácticas de manejo, la fertilización y el sobrepastoreo (Sánchez \& Villaneda, 2009).

El kikuyo es una gramínea tropical de amplia distribución, pero presenta limitantes productivas al verse afectado por las heladas y contener una alta proporción de tejidos de baja digestibilidad, lo que ocasiona una baja respuesta productiva por parte del animal que consume este tipo de pasto (Vargas-Martínez et al., 2018).

Se ha encontrado un buen comportamiento en la calidad y la producción de leche en vacas que pastoreaban en una asociación de Festuca arundinacea y Lotus uliginosus en el trópico alto de Colombia, al compararla con pasturas puras de Cenchrus clandestinus. Sin embargo, no se cuenta con abundante registro de materiales evaluados y liberados para el país, en el caso de asociaciones de gramíneas y leguminosas (Castro-Rincón et al., 2019; Morales et al., 2013).

La introducción y el estudio continuo de nuevas especies de pastos, permite conocer su adaptación a los diferentes medios ecológicos y aumentar la producción de forraje en periodos de tiempo más cortos (CadenaGuerrero et al., 2019b). También, la adaptación de germoplasma a las condiciones de clima, de suelos y a la tolerancia o resistencia a las plagas y patógenos de una región es el punto de partida de cualquier programa de investigación en pastos (Cuesta-Muñoz et al., 2006; Toledo, 1982).

En Colombia, la evaluación de germoplasma forrajero para zonas del trópico alto se suspendió a mediados de los ochenta y con ello la incorporación de nuevos materiales con alta producción y calidad, razón por la cual, 
en estas zonas se usan materiales recomendados hace veinticinco años (Cuesta-Muñoz et al., 2006). Sin embargo, como una estrategia para intensificar la productividad de los sistemas forrajeros, se han introducido algunas gramíneas forrajeras mejoradas, principalmente raigrases diploides y tetraploides y en menor proporción festucas y tréboles, pero sin llegar a un conocimiento profundo de su adaptabilidad y producción en diferentes ambientes del Trópico Alto de Nariño (Cadena-Guerrero et al., 2019a; b; Carulla et al., 2003).

Los estudios tendientes a resolver la problemática antes descrita son limitados y, generalmente se han enfocado a la investigación de casos particulares, con especies introducidas y recomendaciones de manejo proporcionadas por las casas comerciales, sin tener en cuenta las condiciones agroecológicas de la región.

Con base en el régimen de distribución de las lluvias durante el año bajo condiciones tropicales, donde se presentan periodos intermedios de bajas precipitaciones, se hace necesario el aporte de las necesidades hídricas de los forrajes a través de riegos suplementarios. Se deben pretender aguas de buena calidad fisicoquímica y adoptar métodos de riegos presurizados, determinando las necesidades hídricas de los forrajes y suministrándolas a través de sistemas de riego por aspersión, favoreciendo de esta forma la germinación de la semilla y el mantenimiento de la productividad en épocas de escases hídrica (Camacho-García \& García-Muñiz, 2003).

Las deficiencias de fósforo en los suelos ácidos disminuyen los rendimientos y afectan la calidad de muchos cultivos, incluyendo los pastos, por lo que se recomienda corregir los problemas de acidez con rocas fosfóricas o cales, las cuales favorecen: un aumento de la mineralización neta de nitrogeno, una mayor disponibilidad de fósforo en la planta, disminución de la toxicidad del aluminio en el subsuelo, aumento de las concentraciones de molibdeno en la planta, disminución de las concentraciones de magnesio vegetal y aumento en el pH del suelo por encima del control (Cobo, 2003). La estacionalidad de la oferta forrajera es uno de los principales problemas de los sistemas de producción ganadera de la región, por lo tanto, se requiere desarrollar e implementar nuevas alternativas de producción en diferentes ambientes, mediante la evaluación de la adaptación de germoplasma que pueda ser incluido en los sistemas de producción lechera y que garanticen una mejora en la alimentación y nutrición de los animales.

El objetivo de este estudio fue evaluar la interacción de seis mezclas de gramíneas y leguminosas bajo tres niveles de enmienda y dos niveles de riego en el trópico alto de Nariño.

\section{Materiales y métodos}

La investigación se realizó entre diciembre de 2017 y noviembre de 2018, en el centro de investigación Obonuco de la Corporación Colombiana de Investigación Agropecuaria (AGROSAVIA), ubicado en Pasto, Nariño, Colombia; localizada a $1^{\circ} 13^{\prime \prime}$ de latitud norte y $76^{\circ} 16^{\prime \prime}$ de longitud oeste, en un suelo andisol de textura franco arcillosa, $\mathrm{pH}$ : 5,2, materia orgánica (MO): 17,3\%, fósforo disponible (P): $25,08 \mathrm{mg} \mathrm{kg}^{-1}$, azufre disponible (S): 7,62 $\mathrm{mg} \mathrm{kg}^{-1}$, acidez intercambiable $(\mathrm{Al}+\mathrm{H}): 1,88 \mathrm{cmol}_{(+)} \mathrm{kg}^{-1}$, aluminio intercambiable $(\mathrm{Al}): 1,16 \mathrm{cmol}{ }_{(+)} \mathrm{kg}^{-1}$, calcio intercambiable (Ca): 4,41 $\mathrm{cmol}_{(+)} \mathrm{kg}^{-1}$, magnesio intercambiable $(\mathrm{Mg}): 0,12 \mathrm{cmol}_{(+)} \mathrm{kg}^{-1}$, potasio intercambiable $(\mathrm{K}): 0,97 \mathrm{cmol}_{(+)}$ $\mathrm{kg}^{-1}$, sodio intercambiable (Na): 0,32 $\mathrm{cmol}_{(+)} \mathrm{kg}^{-1}$, capacidad de intercambio catiónico (CICE): $8,46 \mathrm{cmol}_{(+)} \mathrm{kg}^{-1}$, arena: $37 \%$, limo: $30 \%$, arcilla: $33 \%$, D: $0,9 \mathrm{~g} \mathrm{~m}^{-3}$, a una altura de $2710 \mathrm{msnm}$, temperatura promedio de $13{ }^{\circ} \mathrm{C}$, humedad relativa de $77 \%$ y precipitación anual de $843 \mathrm{~mm}$ (estación meteorológica automática CI Obonuco).

Se establecieron seis mezclas (Cuadro 1) compuestas por trojan (Lolium perenne L), tetrablend 260 (ryegrass anual tetraploide: $30 \%$; ryegrass híbrido tetraploide: 50\%; pasto azul orchoro: $10 \%$ y trébol rojo: $10 \%$ ), azul orchoro (Dactylis glomerata L), con las leguminosas trébol white gold (Trifolium repens L) y trébol red gold (Trifolium pratense L ); tres niveles de enmienda: nivel 1: $10 \mathrm{t} \mathrm{ha}^{-1} \mathrm{CaMg}\left(\mathrm{CO}_{3}\right)_{2}$, nivel 2: $5 \mathrm{t} \mathrm{ha}^{-1} \mathrm{CaMg}\left(\mathrm{CO}_{3}\right)_{2} \mathrm{y}$ nivel 3: $2 \mathrm{t} \mathrm{ha}^{-1} \mathrm{CaMg}\left(\mathrm{CO}_{3}\right)_{2}$ donde el nivel de 2 se determinó con base a los resultados obtenidos en el análisis de suelos y los siguientes dos niveles se definieron de acuerdo a los ensayos realizados en Nueva Zelanda por Edmeades 
Cuadro 1. Mezcla de forrajes anuales y perennes: trojan (Lolium perenne $\mathrm{L}$ ), tetrablend 260 (mezcla comercial), azul orchoro (Dactylis glomerata $\mathrm{L}$ ), con las leguminosas trébol white gold (Trifolium repens $\mathrm{L}$ ) y trébol red gold (Trifolium pratense $\mathrm{L}$ ) y densidades de siembra $\left(\mathrm{kg} \mathrm{ha}^{-1}\right)$, bajo condiciones del trópico alto de Nariño, Colombia. 2017-2018.

Table 1. Mixtures of annual and perennial forages: trojan (Lolium perennial L.), tetrablend 260 (commercial mixture), orchoro blue (Dactylis glomerata L.), with white gold clover (Trifolium repens L.), and red gold clover (Trifolium pratense L) legumes, and planting densities $\left(\mathrm{kg} \mathrm{ha}^{-1}\right)$, under conditions of the high tropics of Nariño, Colombia. 2017-2018.

\begin{tabular}{|c|c|c|c|c|c|}
\hline Mezclas & Lolium perenne $\mathrm{L}$ & Trifolium repens $\mathrm{L}$ & Trifolium pratense $\mathrm{L}$ & Dactylis glomerata $\mathbf{L}$ & Tetrablend 260 \\
\hline 1 & 28 (trojan) & 3 & - & - & - \\
\hline 2 & 28 (trojan) & - & 6 & - & - \\
\hline 3 & 28 (trojan) & 3 & 6 & - & - \\
\hline 4 & - & 3 & 6 & 25 & - \\
\hline 5 & - & - & - & - & 30 \\
\hline 6 & 28 (trojan) & - & - & - & - \\
\hline
\end{tabular}

Tetrablend 260: (ryegrass anual tetraploide: $30 \%$; ryegrass híbrido tetraploide: $50 \%$; pasto azul orchoro: $10 \%$ y trébol rojo: $10 \%$ ).

et al. (2012). Se usaron dos niveles de riego (con y sin riego), con base en la evapotranspiración de referencia (ETo) del sitio evaluado. Las láminas de agua se aplicaron a través de un riego estacionario sobre ruedas (NUMEDIC), como fuente hídrica se utilizó el agua superficial de una quebrada tipo torrencial. El total de la lámina aplicada fue de 681,71 mm para el periodo evaluado, se realizaron calendarios de riego decadales, los cuales se distribuyeron por época de precipitación de la siguiente forma: para época de altas precipitaciones (marzo, abril, mayo, octubre, noviembre) se aplicó un total de 216,84 mm y para bajas precipitaciones (enero, febrero, junio, julio, agosto y septiembre) se aplicó un total de $464,87 \mathrm{~mm}$.

Los tratamientos aplicados a las unidades experimentales resultaron de la interacción entre los niveles de cada factor, donde el factor mezcla se asignó de manera aleatorizada a las subparcelas (Cuadro 2).

Las variables climáticas se obtuvieron a través de una estación meteorológica automática Vintage Pro-2, la cual se instaló cerca al lote experimental y cuenta con sensores de precipitación, temperatura, brillo solar, humedad relativa, punto de rocío y velocidad del viento, con las cuales se realizó la determinación de la ETo y el cálculo del balance hídrico.

La preparación del terreno se hizo con dos pases de cincel vibratorio y dos pases de rastra pesada, se aplicó cal de acuerdo con los niveles establecidos $\left[10,5\right.$ y 2 tha $\left.^{-1} \mathrm{CaMg}\left(\mathrm{CO}_{3}\right)_{2}\right]$ por unidad experimental y se dejó incorporar por un mes, aproximadamente. La fertilización se realizó manualmente de manera fraccionada, en el momento de la siembra con $50 \mathrm{~kg} \mathrm{P}_{2} \mathrm{O}_{5}$ ha $^{-1}$ y cinco meses después con $150 \mathrm{~kg} \mathrm{~N}^{-1}, 50 \mathrm{~kg} \mathrm{P}_{2} \mathrm{O}_{5}$ ha $^{-1}, 40 \mathrm{~kg} \mathrm{~K}_{2} \mathrm{O} \mathrm{ha}^{-1}, 30 \mathrm{~kg} \mathrm{Mg}$ $\mathrm{ha}^{-1}$ y $30 \mathrm{Kg} \mathrm{S} \mathrm{ha}^{-1}$. Se asignó el factor riego y los tratamientos que contaron con este factor se llevó a cabo teniendo en cuenta el balance hídrico mensual.

Para el experimento se utilizó un diseño de bloques completos al azar con arreglo de parcelas subdivididas, 36 tratamientos, tres repeticiones. Asignando el factor riego a la parcela principal, el factor enmienda con tres niveles a la subparcela y el factor mezcla con seis niveles a la sub-subparcela, El tamaño de la unidad experimental fue de $9 \mathrm{~m}^{2}$, con distancias entre parcelas de $0,3 \mathrm{~m}$, entre calles de $1 \mathrm{~m}$ y distancia entre bloques de $2 \mathrm{~m}$, para un área total de $1677 \mathrm{~m}^{2}$.

A partir del día siete del establecimiento, se realizaron evaluaciones para determinar el número de plantas de trébol (PT), el número de plantas de raigrás (PR), la cobertura de trébol (CT) y la cobertura de raigrás $(\mathrm{CR})$. Luego de tres meses desde la siembra (periodo de establecimiento), se procedió a realizar un corte de uniformización en diferentes tiempos (mezcla 1, 2, 3 y 6: 83 días, mezcla 4: 90 días y mezcla 5: 74 días), donde se evaluaron las variables: cobertura del raigrás $(\mathrm{CR})$, altura $(\mathrm{A})$, número de hojas $(\# \mathrm{H})$ y materia seca $(\mathrm{MS})$, teniendo en cuenta 
Cuadro 2. Tratamientos evaluados con base en mezclas de forrajes anuales y perennes: trojan (Lolium perenne L), tetrablend 260 (mezcla comercial), azul orchoro (Dactylis glomerata L), con las leguminosas trébol white gold (Trifolium repens L) y trébol red gold (Trifolium pratense L), con diferentes niveles de enmienda y dos niveles de riego, bajo condiciones del trópico alto de Nariño, Colombia, 2017-2018.

Table 2. Treatments evaluated based on mixtures of annual and perennial forages: trojan (Lolium perennial L), tetrablend 260 (commercial mixture), orchoro blue (Dactylis glomerata L), with gold white clover (Trifolium repens L), and red gold clover (Trifolium pratense L) legumes, with different levels of amendment and two levels of irrigation, under conditions of the high tropics of Nariño, Colombia, 2017-2018.

\begin{tabular}{|c|c|c|c|}
\hline Trat. & $\begin{array}{l}\text { Leguminosas + forrajes } \\
+\mathrm{t} \mathrm{ha}^{-1} \mathrm{CaMg}\left(\mathrm{CO}_{3}\right)_{2}\end{array}$ & Trat. & $\begin{array}{c}\text { Leguminosas + forrajes } \\
+\mathrm{t} \mathrm{ha}^{-1} \mathrm{CaMg}\left(\mathrm{CO}_{3}\right)_{2}\end{array}$ \\
\hline $\mathrm{T} 1$ & $\mathrm{~T}+\mathrm{Tb}+\mathrm{CR}+10 \mathrm{t} \mathrm{CaMg}\left(\mathrm{CO}_{3}\right)_{2}$ & $\mathrm{~T} 19$ & $\mathrm{~T}+\mathrm{Tb}+\mathrm{SR}+10 \mathrm{t} \mathrm{CaMg}\left(\mathrm{CO}_{3}\right)_{2}$ \\
\hline $\mathrm{T} 2$ & $\mathrm{~T}+\mathrm{Tb}+\mathrm{CR}+5 \mathrm{t} \mathrm{CaMg}\left(\mathrm{CO}_{3}\right)_{2}$ & $\mathrm{~T} 20$ & $\mathrm{~T}+\mathrm{Tb}+\mathrm{SR}+5 \mathrm{t} \mathrm{CaMg}\left(\mathrm{CO}_{3}\right)_{2}$ \\
\hline $\mathrm{T} 3$ & $\mathrm{~T}+\mathrm{Tb}+\mathrm{CR}+2 \mathrm{t} \mathrm{CaMg}\left(\mathrm{CO}_{3}\right)_{2}$ & $\mathrm{~T} 21$ & $\mathrm{~T}+\mathrm{Tb}+\mathrm{SR}+2 \mathrm{t} \mathrm{CaMg}\left(\mathrm{CO}_{3}\right)_{2}$ \\
\hline $\mathrm{T} 4$ & $\mathrm{~T}+\mathrm{Tr}+\mathrm{CR}+10 \mathrm{t} \mathrm{CaMg}\left(\mathrm{CO}_{3}\right)_{2}$ & $\mathrm{~T} 22$ & $\mathrm{~T}+\mathrm{Tr}+\mathrm{SR}+10$ t $\mathrm{CaMg}\left(\mathrm{CO}_{3}\right)_{2}$ \\
\hline $\mathrm{T} 5$ & $\mathrm{~T}+\mathrm{Tr}+\mathrm{CR}+5 \mathrm{t} \mathrm{CaMg}\left(\mathrm{CO}_{3}\right)_{2}$ & $\mathrm{~T} 23$ & $\mathrm{~T}+\mathrm{Tr}+\mathrm{SR}+5 \mathrm{t} \mathrm{CaMg}\left(\mathrm{CO}_{3}\right)_{2}$ \\
\hline T6 & $\mathrm{T}+\mathrm{Tr}+\mathrm{CR}+2 \mathrm{t} \mathrm{CaMg}\left(\mathrm{CO}_{3}\right)_{2}$ & $\mathrm{~T} 24$ & $\mathrm{~T}+\mathrm{Tr}+\mathrm{SR}+2$ t $\mathrm{CaMg}\left(\mathrm{CO}_{3}\right)_{2}$ \\
\hline $\mathrm{T} 7$ & $\mathrm{~T}+\mathrm{Tr}+\mathrm{Tb}+\mathrm{CR}+10 \mathrm{t} \mathrm{CaMg}\left(\mathrm{CO}_{3}\right)_{2}$ & $\mathrm{~T} 25$ & $\mathrm{~T}+\mathrm{Tr}+\mathrm{Tb}+\mathrm{SR}+10 \mathrm{t} \mathrm{CaMg}\left(\mathrm{CO}_{3}\right)_{2}$ \\
\hline $\mathrm{T} 8$ & $\mathrm{~T}+\mathrm{Tr}+\mathrm{Tb}+\mathrm{CR}+5 \mathrm{t} \mathrm{CaMg}\left(\mathrm{CO}_{3}\right)_{2}$ & $\mathrm{~T} 26$ & $\mathrm{~T}+\mathrm{Tr}+\mathrm{Tb}+\mathrm{SR}+5 \mathrm{t} \mathrm{CaMg}\left(\mathrm{CO}_{3}\right)_{2}$ \\
\hline T9 & $\mathrm{T}+\mathrm{Tr}+\mathrm{Tb}+\mathrm{CR}+2 \mathrm{t} \mathrm{CaMg}\left(\mathrm{CO}_{3}\right)_{2}$ & $\mathrm{~T} 27$ & $\mathrm{~T}+\mathrm{Tr}+\mathrm{Tb}+\mathrm{SR}+2 \mathrm{t} \mathrm{CaMg}\left(\mathrm{CO}_{3}\right)_{2}$ \\
\hline $\mathrm{T} 10$ & $\mathrm{Ao}+\mathrm{Tb}+\mathrm{Tr}+\mathrm{CR}+10$ t $\mathrm{CaMg}\left(\mathrm{CO}_{3}\right)_{2}$ & $\mathrm{~T} 28$ & $\mathrm{Ao}+\mathrm{Tb}+\mathrm{Tr}+\mathrm{SR}+10 \mathrm{t} \mathrm{CaMg}\left(\mathrm{CO}_{3}\right)_{2}$ \\
\hline $\mathrm{T} 11$ & $\mathrm{Ao}+\mathrm{Tb}+\mathrm{Tr}+\mathrm{CR}+5 \mathrm{t} \mathrm{CaMg}\left(\mathrm{CO}_{3}\right)_{2}$ & $\mathrm{~T} 29$ & $\mathrm{Ao}+\mathrm{Tb}+\mathrm{Tr}+\mathrm{SR}+5$ t CaMg $\left(\mathrm{CO}_{3}\right)_{2}$ \\
\hline $\mathrm{T} 12$ & $\mathrm{Ao}+\mathrm{Tb}+\mathrm{Tr}+\mathrm{CR}+2 \mathrm{t} \mathrm{CaMg}\left(\mathrm{CO}_{3}\right)_{2}$ & $\mathrm{~T} 30$ & $\mathrm{Ao}+\mathrm{Tb}+\mathrm{Tr}+\mathrm{SR}+2 \mathrm{t} \mathrm{CaMg}\left(\mathrm{CO}_{3}\right)_{2}$ \\
\hline $\mathrm{T} 13$ & $\mathrm{Tt}+\mathrm{CR}+10 \mathrm{t} \mathrm{CaMg}\left(\mathrm{CO}_{3}\right)_{2}$ & $\mathrm{~T} 31$ & $\mathrm{Tt}+\mathrm{SR}+10 \mathrm{t} \mathrm{CaMg}\left(\mathrm{CO}_{3}\right)_{2}$ \\
\hline $\mathrm{T} 14$ & $\mathrm{Tt}+\mathrm{CR}+5 \mathrm{t} \mathrm{CaMg}\left(\mathrm{CO}_{3}\right)_{2}$ & $\mathrm{~T} 32$ & $\mathrm{Tt}+\mathrm{SR}+5 \mathrm{t} \mathrm{CaMg}\left(\mathrm{CO}_{3}\right)_{2}$ \\
\hline $\mathrm{T} 15$ & $\mathrm{Tt}+\mathrm{CR}+2 \mathrm{t} \mathrm{CaMg}\left(\mathrm{CO}_{3}\right)_{2}$ & $\mathrm{~T} 33$ & $\mathrm{Tt}+\mathrm{SR}+2 \mathrm{t} \mathrm{CaMg}\left(\mathrm{CO}_{3}\right)_{2}$ \\
\hline $\mathrm{T} 16$ & $\mathrm{~T}+\mathrm{CR}+10 \mathrm{t} \mathrm{CaMg}\left(\mathrm{CO}_{3}\right)_{2}$ & $\mathrm{~T} 34$ & $\mathrm{~T}+\mathrm{SR}+10 \mathrm{t} \mathrm{CaMg}\left(\mathrm{CO}_{3}\right)_{2}$ \\
\hline $\mathrm{T} 17$ & $\mathrm{~T}+\mathrm{CR}+5 \mathrm{t} \mathrm{CaMg}\left(\mathrm{CO}_{3}\right)_{2}$ & T35 & $\mathrm{T}+\mathrm{SR}+5 \mathrm{t} \mathrm{CaMg}\left(\mathrm{CO}_{3}\right)_{2}$ \\
\hline $\mathrm{T} 18$ & $\mathrm{~T}+\mathrm{CR}+2$ t $\mathrm{CaMg}\left(\mathrm{CO}_{3}\right)_{2}$ & T36 & $\mathrm{T}+\mathrm{SR}+2 \mathrm{t} \mathrm{CaMg}\left(\mathrm{CO}_{3}\right)_{2}$ \\
\hline
\end{tabular}

T: trojan; Tb: trébol white gold; Tr: trébol red gold; Ao: azul orchoro; Tt: tetrablend 260; CR: con riego; SR: sin riego / T: trojan; Tb: white gold clover; Tr: red gold clover; Ao: orchoro blue; Tt: tetrablend 260; CR: with irrigation; SR: no irrigation.

que los materiales sembrados son mezclas de diferentes especies y de acuerdo a la cobertura, estado fisiológico o el conteo de hojas por planta (McCarthy et al., 2016). El corte a las especies evaluadas se realizó a $10 \mathrm{~cm}$ del suelo.

Durante el periodo de producción, cada unidad experimental se dividió en cuatro cuadrantes, los cuales se cosecharon a los 28, 35 y 42, días, se realizaron evaluaciones antes del corte de uniformización de las siguientes variables, de acuerdo con la metodología descrita por Toledo (1982) durante cuatro cortes:

a) Vigor: se empleó una escala de uno a cinco, donde uno corresponde a la escala de mal vigor, dos a vigor débil, tres a vigor intermedio, cuatro a buen vigor y cinco a vigor excelente, esta variable se midió durante el establecimiento.

b) Número de plantas: se contó el número de plantas por $\mathrm{m}^{2}$, usando un marco de $0,25 \mathrm{~m}^{2}$, el cual se ubicó en la parte central de cada unidad experimental.

c) Altura de la planta $(\mathrm{cm})$ : se registró la altura mediante una regla métrica, en cinco plantas seleccionadas al azar en cada unidad experimental. Se midió desde el suelo hasta el punto más alto de la planta, sin estirarla y sin contar la inflorescencia.

d) Cobertura (\%): se midió visualmente en campo con un marco de $0,25 \mathrm{~m}^{2}$, el cual se ubicó al azar en el área útil de la unidad experimental y basado en el porcentaje de la superficie de terreno que está cubierta por vegetación. 
e) Incidencia de plagas (\%): el grado de afectación se midió en un marco de evaluación de $1 \mathrm{~m}^{2}$, con base en la escala de 1 a 5, donde: 1 corresponde a sin daño, 2 a 1-10 \% de las plantas afectadas (p.a.), 3 a 11-25\% p.a., 4 a $26-40 \%$ p.a. y 5 a más del $40 \%$ de p.a.

f) Incidencia de enfermedades (\%): se midió visualmente en campo con base en el porcentaje de incidencia ( 0 a $100 \%$ ) de la enfermedad causada por el inoculo natural en la parcela, así fuera una sola mancha en cada hoja.

g) Severidad de enfermedades: se midió con escala de (0-9) para manchas foliares causadas por Pyricularia grisea en raigrás (Lolium sp.) (ETHzürich, 2016).

h) Deficiencias nutricionales: se determinó comparando el color de las hojas con una escala de 1 a 4 , donde 1 es ausencia de deficiencia, color de follaje y 4 alta deficiencia, planta con clorosis en casi toda el área foliar, coloración roja y púrpura en muchas hojas, necrosis parcial y total normal.

Los datos se analizaron con base en su varianza y distribución normal, las variables cuantitativas se analizaron a través de un ANOVA acompañado de la prueba de comparación de medias de Tukey $(p<0,05)$, las variables cualitativas se analizaron a través de tablas de contingencia acompañadas de la prueba de Chi-cuadrado de Person $(\mathrm{p} \leq 0,05)$. La información obtenida se analizó a través del Software R V.3.6 (R Development Core Team 3.0.1., 2013), con los paquetes agricolae (de Mendiburu, 2017) y ggplot2 (Wickham, 2016).

\section{Resultados}

\section{Periodo de establecimiento}

Para la fase de establecimiento no se observaron efectos asociados a la interacción de factores riego, enmienda y mezcla $(p>0,05)$. Para el factor mezcla se observaron diferencias significativas (Cuadro 3). En cuanto a la variable número de plantas de trébol (PT) y cobertura de trébol $(\mathrm{CT})$, los mayores valores los presentó la mezcla 4, con 14,17 y $35,38 \%$, respectivamente. El mayor porcentaje de CR lo mostró la mezcla 2 con 74,80 \%, siendo consecuentes con: PR de 57,80 .

Cuadro 3. Variables agronómicas de seis mezclas forrajeras: trojan (Lolium perenne L.), tetrablend 260 (mezcla comercial), azul orchoro (Dactylis glomerata L.), con las leguminosas trébol white gold (Trifolium repens L.) y trébol red gold (Trifolium pratense L.), evaluadas en la fase de establecimiento en Pasto, Nariño, Colombia. 2017- 2018.

Table 3. Agronomic variables of six fodder mixtures: trojan (Lolium perennial L.),tetrablend 260 (commercial mixture), orchoro blue (Dactylis glomerata L.), with white gold clover (Trifolium repens L.), and red gold clover (Trifolium pratense) legumes, evaluated in the establishment phase in Pasto, Nariño, Colombia. 2017-2018.

\begin{tabular}{lcccc}
\hline Mezclas & PT & PR & CT $(\%)$ & CR $(\%)$ \\
\hline Trojan, trébol blanco & $6,71^{\mathrm{b}}$ & $53,99^{\mathrm{ab}}$ & $16,94^{\mathrm{b}}$ & $72,89^{\mathrm{a}}$ \\
Trojan, trébol rojo & $9,48^{\mathrm{b}}$ & $57,80^{\mathrm{a}}$ & $29,13^{\mathrm{a}}$ & $74,80^{\mathrm{a}}$ \\
Trojan, trébol rojo, trébol blanco & $9,49^{\mathrm{b}}$ & $56,61^{\mathrm{a}}$ & $29,83^{\mathrm{a}}$ & $74,03^{\mathrm{a}}$ \\
Azul orchoro, trébol blanco, trébol rojo & $14,17^{\mathrm{a}}$ & $46,02^{\mathrm{bc}}$ & $35,38^{\mathrm{a}}$ & $62,11^{\mathrm{ab}}$ \\
Tetrablend 260 & $2,51^{\mathrm{c}}$ & $40,82^{\mathrm{c}}$ & $6,01 \mathrm{~b}^{\mathrm{c}}$ & $57,97^{\mathrm{b}}$ \\
Trojan & $0,00^{\mathrm{c}}$ & $54,19^{\mathrm{ab}}$ & $0,00^{\mathrm{c}}$ & $72,83^{\mathrm{a}}$ \\
EE \pm & 0,89 & 2,5 & 3,06 & 3,37 \\
\hline
\end{tabular}

a,b,c medias con letras diferentes dentro de una misma columna difieren entre sí, según la prueba de Tukey (p $\leq 0,05)$. PT: número de plantas de trébol, PR: número de plantas de raigrás, CT: cobertura de trébol, CR: cobertura de raigrás / a,b,c averages with different letters within the same column differ from each other, according to Tukey's test ( $\mathrm{p} \leq 0.05)$. PT: number of clover plants, PR: number of ryegrass plants, CT: clover cover, CR: ryegrass cover. 
Para cobertura de arvenses [(corazón herido (Polygonum nepalense Meins), lengua de vaca (Rumex crispus), nabo forrajero (Brassica napus), y verbena (Verbena officinalis)] se pudo evidenciar que no se presentaron diferencias estadísticas para las interacciones de tres y dos factores, ni para los factores principales por separado.

\section{Época de altas precipitaciones}

Para la edad de 28 días (Cuadro 4), no se observó efecto de la interacción de los factores evaluados (p>0,05). Por lo tanto, se analizó el efecto de los factores principales por separado, donde el factor mezcla presento diferencias significativas en las variables evaluadas $(\mathrm{p}<0,05)$.

Cuadro 4. Rendimiento y variables agronómicas de seis mezclas forrajeras: trojan (Lolium perenne L.), tetrablend 260 (mezcla comercial), azul orchoro (Dactylis glomerata L.), con las leguminosas trébol white gold (Trifolium repens L.) y trébol red gold (Trifolium pratense L), evaluadas en época de altas precipitaciones, a la edad de 28 días, en Pasto, Nariño, Colombia. $2017-2018$.

Table 4. Yield and agronomic variables of six fodder mixtures: trojan (Lolium perennial L.), tetrablend 260 (commercial mixture), orchoro blue (Dactylis glomerata L.), with white gold clover (Trifolium repens L.), and red gold clover (Trifolium pratense) legumes, evaluated during high rainfall, at the age of 28 days, in Pasto, Nariño, Colombia. 2017-2018.

\begin{tabular}{|c|c|c|c|c|c|}
\hline Mezclas & CT $(\%)$ & CR $(\%)$ & $\mathrm{A}(\mathbf{c m})$ & $\# \mathbf{H}$ & MS (kg ha $\left.{ }^{-1}\right)$ \\
\hline Trojan, trébol blanco & $33,61^{\mathrm{c}}$ & $96,67^{\mathrm{a}}$ & $25,67^{\text {bc }}$ & $1,47^{\mathrm{a}}$ & $1853,50^{\mathrm{b}}$ \\
\hline Trojan, trébol rojo & $53,89^{b}$ & $97,94^{\mathrm{a}}$ & $27,89^{\mathrm{bc}}$ & $1,53^{\mathrm{a}}$ & $1923,89^{b}$ \\
\hline Trojan, trébol rojo, trébol blanco & $58,89^{\mathrm{b}}$ & $99,44^{\mathrm{a}}$ & $28,78^{\mathrm{b}}$ & $1,47^{\mathrm{a}}$ & $2082,89^{b}$ \\
\hline Azul orchoro, trébol blanco, trébol rojo & $100,00^{\mathrm{a}}$ & $98,33^{\mathrm{a}}$ & $27,94^{\mathrm{bc}}$ & $1,05^{\mathrm{b}}$ & $3363,44^{\mathrm{a}}$ \\
\hline Tetrablend 260 & $26,00^{\mathrm{c}}$ & $100,00^{\mathrm{a}}$ & $33,06^{\mathrm{a}}$ & $1,36^{\mathrm{a}}$ & $1954,00^{\mathrm{b}}$ \\
\hline Trojan & $0,00^{\mathrm{d}}$ & $97,78^{\mathrm{a}}$ & $25,22^{\mathrm{c}}$ & $1,47^{\mathrm{a}}$ & $1731,18^{\mathrm{b}}$ \\
\hline $\mathrm{EE} \pm$ & 3,45 & 1,07 & 0,8 & 0,09 & 0,092 \\
\hline
\end{tabular}

a,b,c medias con letras diferentes dentro de una misma columna difieren entre sí, según la prueba de Tukey ( $\leq 0,05)$. PT: $\mathrm{N}^{\mathrm{o}}$ de plantas de trébol, PR: No. de plantas de raigrás, CT: cobertura de trébol, CR: cobertura de raigras / a,b,c averages with different letters within the same column differ from each other, according to Tukey's test $(\mathrm{p} \leq 0.05)$; PT: number of clover plants, PR: number of rye plants, CT: clover cover, CR: rye coverage.

Se encontraron diferencias en las variables cobertura de trébol (CT), en donde se evidenció un mayor valor para la mezcla $4(100 \%)$ con respecto a las demás mezclas. De igual manera para la altura (A), en donde la mezcla 5 superó a todas las demás mezclas $(33,06 \mathrm{~cm})$. Con respecto al número de hojas (\# H), la mezcla 2 mostró mayor valor $(1,53)$. Para la variable MS, la mezcla 4 superó a las demás mezclas con una producción de 3363,44 kg ha' ${ }^{1}$.

Para las variables de adaptación no se observaron diferencias ( $\mathrm{p}>0,05)$, el vigor se mantuvo entre bueno y excelente; no se manifestó deficiencia nutricional en la mayoría de los tratamientos. Si hubo diferencias $(\mathrm{p}>0,05)$ en las variables incidencia de plagas, severidad a enfermedades e incidencia de enfermedades. La incidencia de plagas se mantuvo baja a los 28 días de edad, presentándose valores de 0 a 1 según la escala de evaluación.

Con respecto a severidad de enfermedades el 67,6\% de los tratamientos no mostraron síntomas de enfermedades y el $32 \%$ de los tratamientos presentó el valor de 1 según la escala de evaluación.

Para la edad de 35 días no se presentaron diferencias estadísticas entre las interacciones de tres y dos factores, por lo cual se analizó el efecto de los factores principales por separado, donde el factor mezcla presentó diferencias significativas (Cuadro 5). Se observaron diferencias significativas en las variables altura (A), número de hojas (\# H) y rendimiento de MS. En cuanto a la variable A, el mayor valor lo presentó la mezcla 5 (M5) con 47,89 cm, seguida 
Cuadro 5. Rendimiento y variables agronómicas de seis mezclas forrajeras: trojan (Lolium perenne L.), tetrablend 260 (mezcla comercial), azul orchoro (Dactylis glomerata L.), con las leguminosas trébol white gold (Trifolium repens L.) y trébol red gold (Trifolium pratense L., evaluadas en época de altas precipitaciones, a la edad de 35 días, en Pasto, Nariño, Colombia. 2017 - 2018.

Table 5. Yield and agronomic variables of six fodder mixtures: trojan (Lolium perennial L.), tetrablend 260 (commercial mixture), orchoro blue (Dactylis glomerata L.), with white gold clover (Trifolium repens L.), and red gold clover (Trifolium pratense) legumes evaluated during high rainfall, at the age of 35 days, in Pasto, Nariño, Colombia. 2017-2018.

\begin{tabular}{|c|c|c|c|c|}
\hline Mezclas & CR $(\%)$ & $\mathbf{A}(\mathbf{c m})$ & $\# \mathbf{H}$ & MS (kg ha-1) \\
\hline Trojan, trébol blanco & $97,78^{\mathrm{a}}$ & $22,28^{\mathrm{c}}$ & $1,80^{\mathrm{c}}$ & $2555,17^{\mathrm{bc}}$ \\
\hline Trojan, trébol rojo & $97,22^{\mathrm{a}}$ & $24,17^{\mathrm{c}}$ & $1,94^{\mathrm{bc}}$ & $2604,00^{\mathrm{b}}$ \\
\hline Trojan, trébol rojo, trébol blanco & $100^{\mathrm{a}}$ & $27,22^{\mathrm{c}}$ & $1,86^{\mathrm{c}}$ & $2596,17^{b}$ \\
\hline Azul orchoro, trébol blanco, trébol rojo & $97,78^{\text {a }}$ & $39,94^{\mathrm{b}}$ & $2,53^{\mathrm{a}}$ & $3754,61^{\mathrm{a}}$ \\
\hline Tetrablend 260 & $100^{\mathrm{a}}$ & $47,89^{\mathrm{a}}$ & $2,39^{\mathrm{ab}}$ & $2662,44^{\mathrm{b}}$ \\
\hline Trojan & $97,22^{\mathrm{a}}$ & $24,11^{\mathrm{c}}$ & $2,00^{\mathrm{bc}}$ & $2086,44^{\mathrm{c}}$ \\
\hline $\mathrm{EE} \pm$ & 2,58 & 1,34 & 0,11 & 0,12 \\
\hline
\end{tabular}

a,b,c medias con letras diferentes dentro de una misma columna difieren entre sí, según la prueba de Tukey $(p \leq 0,05)$ / a,b,c averages with different letters within the same column differ from each other, according to Tukey's test ( $\mathrm{p} \leq 0.05)$. CR: cobertura de raigrás; A: altura; \# H: número de hojas; MS: materia seca / CR: ryegrass coverage; A: height; \# H: number of leaves; MS: dry matter.

de la mezcla 4 (M4) con 39,94 cm. Los resultados de las variables \# H y rendimiento de MS de la M4 fueron los más altos con 2,53 y $3754,61 \mathrm{~kg} \mathrm{ha}^{-1}$, respectivamente.

Las diferencias significativas ocurridas en los tratamientos para la variable cobertura de trébol se muestran en la Figura 1. La mayor cobertura se observó en aquellos tratamientos conformados por la gramínea azul orchoro (Dactylis glomerata $\mathrm{L}$ ), con y sin riego bajo los diferentes niveles de enmienda $\left[2,5\right.$ y $10 \mathrm{t}$ de $\left.\mathrm{CaMg}\left(\mathrm{CO}_{3}\right)_{2}\right]$.
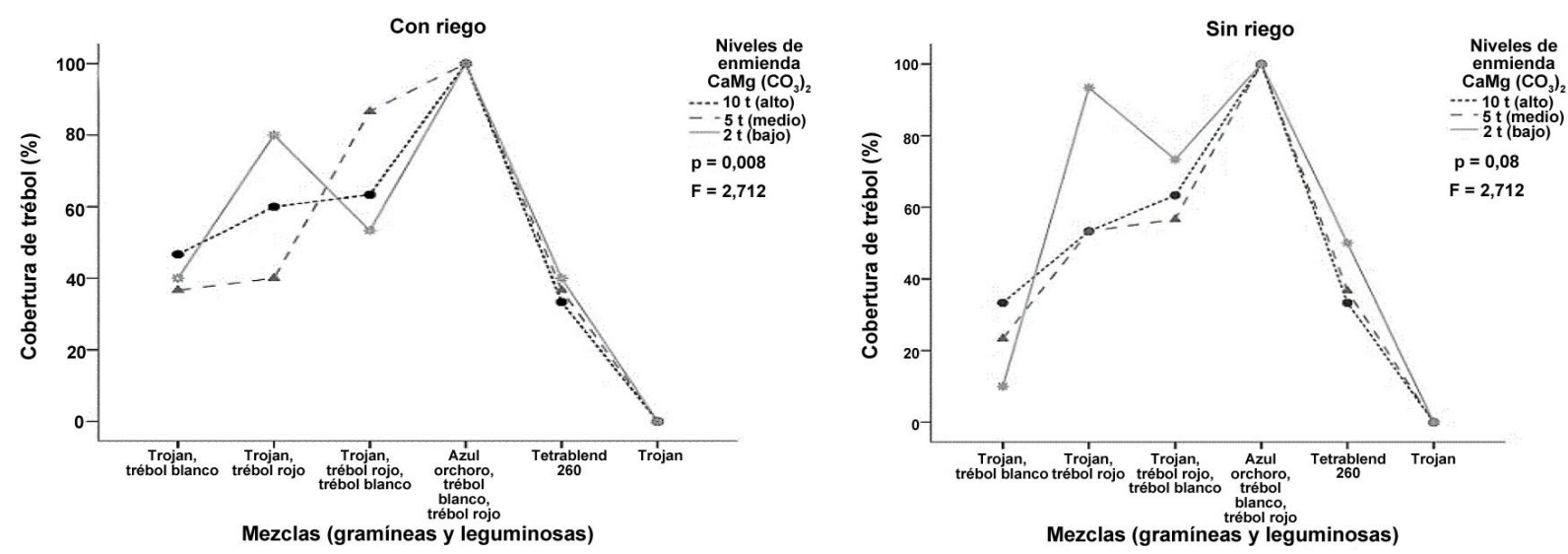

Figura 1. Cobertura de trébol white gold (Trifolium repens L.) y trébol red gold (Trifolium pratense L.), con y sin riego a la edad de 35 días. Pasto, Nariño, Colombia. 2017-2018.

Figure 1. White gold clover (Trifolium repens L.) and red gold clover (Trifolium pratense L.) coverage, with and without irrigation at the age of 35 days. Pasto, Nariño, Colombia. 2017-2018.

Las variables vigor, incidencia de plagas y severidad de enfermedades de los 36 tratamientos evaluados a la edad de 35 días presentaron diferencias significativas $(\mathrm{p}<00,5)$, a diferencia de las variables incidencia de 
enfermedades y deficiencia nutricional que parecen no haber sido influenciadas por los tratamientos. El vigor se mantuvo en una escala excelente para el 85,2 \% de los tratamientos, contrario al 14,8 \% ubicado en una escala de bueno. En cuanto a la incidencia de plagas el $97,2 \%$ de los tratamientos no presentaron daños, solo el 2,8 \% tuvieron afecciones.

Para la variable severidad de enfermedades el 50,9\% de los tratamientos no presentaron ningún síntoma y el $49,1 \%$ mostraron de 1 a $5 \%$ del área foliar infectada.

Para la edad de 42 días, no se presentaron diferencias estadísticas entre las interacciones de tres y dos factores, por lo cual, se observa el efecto de los factores principales por separado, donde el factor mezcla presentó diferencias significativas en las variables cobertura de trébol (CT), altura, número de hojas (H) y rendimiento en materia seca (Cuadro 6). En cuanto a la variable CT el mayor valor lo obtuvo la mezcla 5 con 99,44\%; para la variable A el mayor valor lo presentó la mezcla 5 con 59,50 cm, seguida de la mezcla 4 con 42,72 cm (Cuadro 6). Los resultados de las variables número de hojas y rendimiento de materia seca de la mezcla 4 fueron los más altos con 3,05 y $4708,72 \mathrm{~kg} \mathrm{ha}^{-1}$, respectivamente (Cuadro 6).

Cuadro 6. Rendimiento y variables agronómicas de seis mezclas forrajeras: trojan (Lolium perenne L.), tetrablend 260 (mezcla comercial), azul orchoro (Dactylis glomerata L.), con las leguminosas trébol white gold (Trifolium repens L.) y trébol red gold (Trifolium pratense L), evaluadas en época de altas precipitaciones, a la edad de 42 días, en Pasto, Nariño, Colombia. 2017 - 2018.

Table 6. Yield and agronomic variables of six fodder mixtures: trojan (Lolium perennial L), tetrablend 260 (commercial mixture), orchoro blue (Dactylis glomerata L.), with white gold clover (Trifolium repens L.), and red gold clover (Trifolium pratense) legumes, evaluated during high rainfall, at the age of 42 days, in Pasto, Nariño, Colombia. 2017-2018.

\begin{tabular}{|c|c|c|c|c|c|}
\hline Mezclas & $\mathrm{CT}(\%)$ & CR $(\%)$ & $\mathrm{A}(\mathrm{cm})$ & $\# \mathbf{H}$ & MS (kg ha-1) \\
\hline Trojan, trébol blanco & $41,67^{c}$ & $99^{\mathrm{a}}$ & $30,39^{\mathrm{de}}$ & $2,39^{\mathrm{b}}$ & $3051,61^{\mathrm{bc}}$ \\
\hline Trojan, trébol rojo & $83,89^{\mathrm{b}}$ & $100^{\mathrm{a}}$ & $32,28^{\mathrm{cd}}$ & $2,50^{\mathrm{b}}$ & $3433,89^{\mathrm{bc}}$ \\
\hline Trojan, trébol rojo, trébol blanco & $85,56^{\mathrm{b}}$ & $97,22^{\mathrm{a}}$ & $35,33^{\mathrm{c}}$ & $2,44^{\mathrm{b}}$ & $3291,67^{\mathrm{bc}}$ \\
\hline Azul orchoro, trébol blanco, trébol rojo & $99,44^{\mathrm{a}}$ & $100^{\mathrm{a}}$ & $42,72^{\mathrm{b}}$ & $3,05^{\mathrm{a}}$ & $4708,72^{\mathrm{a}}$ \\
\hline Tetrablend 260 & $43,33^{\mathrm{c}}$ & $100^{\mathrm{a}}$ & $59,50^{\mathrm{a}}$ & $2,47^{\mathrm{b}}$ & $3788,22^{\mathrm{b}}$ \\
\hline Trojan & $0,00^{\mathrm{d}}$ & $99,44^{\mathrm{a}}$ & $27,06^{\mathrm{e}}$ & $2,39^{\mathrm{b}}$ & $2874,39^{c}$ \\
\hline $\mathrm{EE} \pm$ & 3,27 & 0,67 & 1,18 & 0,12 & 0,18 \\
\hline
\end{tabular}

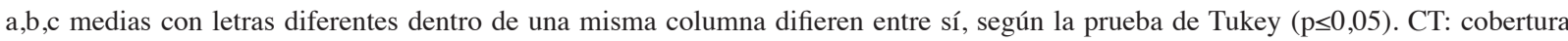
de trébol; CR: cobertura de raigrás; A: altura; \# H: número de hojas; MS: materia seca / a,b,c averages with different letters within the same column differ from each other, according to Tukey's test $(\mathrm{p} \leq 0.05)$; CT: clover coverage; CR: ryegrass coverage; A: height; \# H: number of leaves; MS: dry matter.

Las variables vigor, incidencia de plagas, severidad de enfermedades y deficiencia nutricional de los 36 tratamientos evaluados a la edad de 45 días, presentaron diferencias significativas $(\mathrm{p}<0,5)$, lo observado en estas variables se debe en gran medida al efecto de los tratamientos. El vigor se mantuvo en una escala excelente para el $83,3 \%$ de los tratamientos, contrario al $16,7 \%$ que se mantuvo en una escala de bueno. No se identificó un porcentaje alto de ataque de plagas, ya que la mayoría de los tratamientos (88\%) se mantuvieron en una escala de 1 a $10 \%$ de las plantas afectadas por plagas. La variable severidad de enfermedades presentó un comportamiento similar donde la mayoría de los tratamientos (96\%) se mantuvieron en una escala de 1 a $5 \%$ del área foliar infectada. La mayoría de los tratamientos (95\%) no presentaron deficiencias nutricionales. 


\section{Época de bajas precipitaciones}

Para la edad de 28 días no se presentaron diferencias estadísticas entre las interacciones de tres y dos factores, por lo cual se analiza el efecto de los factores principales por separado, donde el factor mezcla presentó diferencias significativas $(\mathrm{p}<0,05)$.

En el Cuadro 7, se observan diferencias para las variables cobertura de trébol (CT), cobertura de raigrás (CR), altura, número de hojas (H) y materia seca (MS). Con respecto a la A, el Tt 260 superó a las demás mezclas. El \#H fue superior para la mezcla 4 y el contenido de materia seca para esta época fue mayor para la mezcla 1.

Cuadro 7. Rendimiento y variables agronómicas de seis mezclas forrajeras: trojan (Lolium perenne L.), tetrablend 260 (mezcla comercial), azul orchoro (Dactylis glomerata L.), con las leguminosas trébol white gold (Trifolium repens L.) y trébol red gold (Trifolium pratense L), evaluadas en época de bajas precipitaciones, a la edad de 28 días, en Pasto, Nariño, Colombia. 2017 - 2018.

Table 7. Yield and agronomic variables of six fodder mixtures: trojan (Lolium perennial L.), tetrablend 260 (commercial mixture), orchoro blue (Dactylis glomerata L.), with white gold clover (Trifolium repens L.), and red gold clover (Trifolium pratense) legumes, evaluated in times of low rainfall, at the age of 28 days, in Pasto, Nariño, Colombia. 2017-2018.

\begin{tabular}{|c|c|c|c|c|c|}
\hline Mezclas & $\mathrm{CT}(\%)$ & CR $(\%)$ & $\mathbf{A}(\mathbf{c m})$ & $\# \mathbf{H}$ & $\operatorname{MS}\left(\mathbf{k g ~ h a}^{-1}\right)$ \\
\hline Trojan, trébol blanco & $65,6^{b}$ & $93,33^{\mathrm{a}}$ & $27,5^{\mathrm{b}}$ & $1,52^{\mathrm{b}}$ & $2068,27^{\mathrm{a}}$ \\
\hline Trojan, trébol rojo & $80,56^{\mathrm{a}}$ & $90,56^{\mathrm{ab}}$ & $28,22^{\mathrm{b}}$ & $1,63^{\mathrm{b}}$ & $1876,88^{\mathrm{a}}$ \\
\hline Trojan, trébol rojo, trébol blanco & $85,56^{\mathrm{a}}$ & $93,89^{\mathrm{a}}$ & $28,17^{\mathrm{b}}$ & $1,66^{\mathrm{b}}$ & $1908,94^{\mathrm{a}}$ \\
\hline Azul orchoro, trébol blanco, trébol rojo & $79,44^{\mathrm{a}}$ & $80,56^{\mathrm{c}}$ & $26,33^{\text {bc }}$ & $4,52^{\mathrm{a}}$ & $1383,00^{\mathrm{c}}$ \\
\hline Tetrablend 260 & $49,72^{\mathrm{c}}$ & $92,22^{\mathrm{a}}$ & $39,7^{\mathrm{a}}$ & $1,88^{\mathrm{b}}$ & $1806,16^{\mathrm{ab}}$ \\
\hline Trojan & $0,00^{\mathrm{d}}$ & $85^{\mathrm{bc}}$ & $23,39^{\mathrm{c}}$ & $1,55^{\mathrm{b}}$ & $1533,55^{\mathrm{bc}}$ \\
\hline $\mathrm{EE} \pm$ & 2,89 & 1,57 & 0,91 & 0,74 & 0,07 \\
\hline
\end{tabular}

a,b,c medias con letras diferentes dentro de una misma columna difieren entre sí, según la prueba de Tukey (p $\leq 0,05)$. CT: cobertura de trébol; CR: cobertura de raigrás; A: altura; \# H: número de hojas; MS: materia seca / a,b,c averages with different letters within the same column differ from each other, according to Tukey's test ( $\mathrm{p} \leq 0.05)$; CT: clover coverage; CR: ryegrass coverage; A: height; \# H: number of leaves; MS: dry matter.

Las variables, incidencia de plagas, severidad de enfermedades, incidencia de enfermedades y deficiencia nutricional de los 36 tratamientos evaluados a la edad de 28 días presentaron diferencias significativas $(\mathrm{p}<00,5)$, a diferencia de la variable vigor que parece no haber sido influenciada por los tratamientos, ya que se observó que solo el $14 \%$ de los tratamientos presentó un vigor bueno y el $86 \%$ restante presentó un vigor excelente. La incidencia de plagas se mantuvo entre 1 y 2 . La severidad a enfermedades se mantuvo en la escala de evaluación entre 0 y 1 , el $19 \%$ de los tratamientos presentó entre el 1 y $5 \%$ del área foliar infectada. Para la variable incidencia a enfermedades el $19 \%$ de los tratamientos presentó una incidencia del $10 \%$. Solo el 8,33\% de los tratamientos manifestó una incidencia leve y en el 91,66\% de los tratamientos no manifestaron deficiencias nutricionales.

Para la edad de 35 días no se presentaron diferencias estadísticas entre las interacciones de tres y dos factores, por lo cual, se analizó el efecto de los factores principales por separado, donde el factor mezcla presentó diferencias significativas (Cuadro 8), para las variables CT, A, H y MS ( $\left.\mathrm{kg} \mathrm{ha}^{-1}\right)$. Se destacó la mezcla 4 con los mejores valores de CT $(98,89 \%)$ y H $(2,83)$, el Tetrablend 260 mostró la mayor altura con 45,33 cm y la mezcla 3 mostró el mejor rendimiento de MS con $2269 \mathrm{~kg} \mathrm{ha}^{-1}$.

Las variables vigor, severidad de enfermedades, incidencia de enfermedades y deficiencia nutricional a la edad de 35 días de evaluación, presentaron diferencias significativas $(\mathrm{p}<0,05)$, a diferencia de la variable incidencia de 
Cuadro 8. Rendimiento y variables agronómicas de seis mezclas forrajeras: trojan (Lolium perenne L.), tetrablend 260 (mezcla comercial), azul orchoro (Dactylis glomerata L.), con las leguminosas trébol white gold (Trifolium repens L.) y trébol red gold (Trifolium pratense L), evaluadas en época de bajas precipitaciones, a la edad de 35 días, en Pasto, Nariño, Colombia. $2017-2018$.

Table 8. Yield and agronomic variables of six fodder mixtures: trojan (Lolium perennial L.), tetrablend 260 (commercial mixture), orchoro blue (Dactylis glomerata L.), with white gold clover (Trifolium repens L.), and red gold clover (Trifolium pratense) legumes, evaluated in times of low rainfall, at the age of 35 days, in Pasto, Nariño, Colombia. 2017-2018.

\begin{tabular}{lccccc}
\hline Mezclas & CT $(\%)$ & CR $(\%)$ & A $(\mathbf{c m})$ & H & MS $(\mathbf{k g ~ h a})$ \\
\hline Trojan, trébol blanco & $65,28^{\mathrm{b}}$ & $97,78^{\mathrm{a}}$ & $32,28^{\mathrm{b}}$ & $2,02^{\mathrm{bc}}$ & $1993,16^{\mathrm{a}}$ \\
Trojan, trébol rojo & $98,33^{\mathrm{a}}$ & $98,33^{\mathrm{a}}$ & $34^{\mathrm{b}}$ & $2,05^{\mathrm{bc}}$ & $2091,33^{\mathrm{a}}$ \\
Trojan, trébol rojo, trébol blanco & $96,67^{\mathrm{a}}$ & $98,33^{\mathrm{a}}$ & $35,11^{\mathrm{b}}$ & $2,13^{\mathrm{b}}$ & $2269,61^{\mathrm{a}}$ \\
Azul orchoro, trébol blanco, trébol rojo & $98,89^{\mathrm{a}}$ & $95^{\mathrm{a}}$ & $31,89^{\mathrm{b}}$ & $2,83^{\mathrm{a}}$ & $1935,27^{\mathrm{ab}}$ \\
Tetrablend 260 & $60,56^{\mathrm{b}}$ & $97,78^{\mathrm{a}}$ & $45,33^{\mathrm{a}}$ & $2,16^{\mathrm{b}}$ & $2005,11^{\mathrm{a}}$ \\
Trojan & $0,00^{\mathrm{c}}$ & $95^{\mathrm{a}}$ & $25,94^{\mathrm{c}}$ & $1,86^{\mathrm{c}}$ & $1537,83^{\mathrm{b}}$ \\
EE \pm & 3,19 & 0,90 & 0,88 & 0,06 & 0,09 \\
\hline
\end{tabular}

a,b,c medias con letras diferentes dentro de una misma columna difieren entre sí, según la prueba de Tukey ( $\mathrm{p} \leq 0,05)$. CT: cobertura de trébol; CR: cobertura de raigrás; A: altura; H: número de hojas; MS: materia seca / a,b,c averages with different letters within the same column differ from each other, according to Tukey's test ( $\mathrm{p} \leq 0.05)$; CT: clover coverage; CR: ryegrass coverage; A: height; H: number of leaves; MS: dry matter.

plagas que parece no haber sido influenciada por los tratamientos. El vigor se mantuvo en una escala excelente para el $77,78 \%$ de los tratamientos, contrario al $22,22 \%$ que se mantuvo en una escala de bueno. Para la variable severidad de enfermedades el 63,89\% tuvieron del 1 al $5 \%$ del área foliar infectada y el 36,11\% no presentaron ningún síntoma, con una escala de incidencia de un de un $20 \%$ para el 13,88 \% de los tratamientos, $10 \%$ para el $33,33 \%$ y de $0 \%$ para el $52,79 \%$. En cuanto a la deficiencia nutricional un gran porcentaje $(77,78 \%)$ de los tratamientos se mantuvieron en ausencia de deficiencia y el 22,22\% presentaron una deficiencia leve.

Para la edad de 42 días, no se presentaron diferencias estadísticas entre las interacciones de tres y dos factores, por lo cual, se analizó el efecto de los factores principales por separado, donde el factor mezcla presentó diferencias significativas (Cuadro 9), para las variables CT, CR, A, H y MS ( $\left.\mathrm{kg} \mathrm{ha}^{-1}\right)$. Se destacó la mezcla 3 con los mejores valores de CT (100 \%) y CR (99,4 \%), la mezcla 4 presentó el mayor número de hojas con 3,39, la mezcla 3 mostró el mejor rendimiento de MS con $3399 \mathrm{~kg} \mathrm{ha}^{-1}$ y la mezcla 5 la mayor altura con 59,44 cm.

Las variables vigor, severidad de enfermedades, incidencia de enfermedades y deficiencia nutricional, a la edad de 42 días de evaluación, presentaron diferencias significativas $(\mathrm{p}<0,05)$, a diferencia de la variable incidencia de plagas que no la influenciaron los tratamientos. El vigor, se mantuvo en una escala excelente para el $64 \%$ de los tratamientos, contrario al $36 \%$ que se mantuvo en una escala de bueno. Para la variable severidad de enfermedades, el $80 \%$ de los tratamientos no tuvo ningún síntoma de la enfermedad, contrario al $20 \%$ de los tratamientos que presentaron del 1 al $5 \%$ del área foliar infectada. Para la variable incidencia de enfermedades el $67 \%$ de los tratamientos presentaron una incidencia del $0 \%$, el $19 \%$ de tratamientos una incidencia del 10\%, el $5 \%$ de tratamientos una incidencia del $20 \%$ y el $9 \%$ de los tratamientos una incidencia del $30 \%$. En cuanto a la deficiencia nutricional el $64 \%$ de los tratamientos se mantuvieron en ausencia de deficiencia y el $36 \%$ presentaron una deficiencia leve. 
Cuadro 9. Rendimiento y variables agronómicas de seis mezclas forrajeras: trojan (Lolium perenne L.), tetrablend 260 (mezcla comercial), azul orchoro (Dactylis glomerata L), con las leguminosas trébol white (Trifolium repens L.) y trébol red gold (Trifolium pratense L), evaluadas en época de bajas precipitaciones, a la edad de 42 días, en Pasto, Nariño, Colombia. 2017- 2018.

Table 9. Yield and agronomic variables of six fodder mixtures: trojan (Lolium perennial L.), tetrablend 260 (commercial mixture), orchoro blue (Dactylis glomerata L), with white gold clover (Trifolium repens L.), and red gold clover (Trifolium pratense) legumes, evaluated in times of low rainfall, at the age of 42 days, in Pasto, Nariño, Colombia. 2017-2018.

\begin{tabular}{|c|c|c|c|c|c|}
\hline Mezclas & CT $(\%)$ & CR $(\%)$ & $\mathbf{A}(\mathbf{c m})$ & $\mathbf{H}$ & Ms $\left(\mathrm{kg} \mathrm{ha}^{-1}\right)$ \\
\hline Trojan, trébol blanco & $73,3^{b}$ & $98,33^{\mathrm{ab}}$ & $33,78^{\mathrm{c}}$ & $2,47^{\mathrm{c}}$ & $3131,17^{\mathrm{a}}$ \\
\hline Trojan, trébol rojo & $100^{\mathrm{a}}$ & $96,11^{\mathrm{ab}}$ & $37,17^{\mathrm{c}}$ & $2,66^{c}$ & $3379,22^{\mathrm{a}}$ \\
\hline Trojan, trébol rojo, trébol blanco & $100^{\mathrm{a}}$ & $99,44^{\mathrm{a}}$ & $37,44^{\mathrm{c}}$ & $2,75^{\text {bc }}$ & $3399,17^{\mathrm{a}}$ \\
\hline Azul orchoro, trébol blanco, trébol rojo & $100^{\mathrm{a}}$ & $91,11^{\mathrm{b}}$ & $44,78^{\mathrm{b}}$ & $3,39^{\mathrm{a}}$ & $2318,55^{\mathrm{b}}$ \\
\hline Tetrablend 260 & $58,61^{\mathrm{c}}$ & $96,11^{\mathrm{ab}}$ & $59,44^{\mathrm{a}}$ & $2,97^{\mathrm{b}}$ & $2421,16^{\mathrm{b}}$ \\
\hline Trojan & $0,00^{\mathrm{d}}$ & $90,83^{\mathrm{b}}$ & $28,56^{\mathrm{d}}$ & $2,11^{\mathrm{d}}$ & $2265,27^{\mathrm{b}}$ \\
\hline $\mathrm{EE} \pm$ & 2,58 & 1,89 & 1,01 & 0,07 & 0,10 \\
\hline
\end{tabular}

a,b,c medias con letras diferentes dentro de una misma columna difieren entre sí, según la prueba de Tukey $(\mathrm{p} \leq 0,05)$. CT: cobertura de trébol; CR: cobertura de raigrás; A: altura; H: número de hojas; MS: materia seca / a,b,c averages with different letters in the same column differ from each other, according to Tukey's test ( $\mathrm{p} \leq 0.05)$; CT: clover coverage; CR: ryegrass coverage; A: height; H: number of leaves; MS: dry matter.

\section{Discusión}

En siete asociaciones de ovillo, ballico perenne y trébol blanco (Trifolium repens L), se manejó una densidad de siembra de 70-20-10 en donde se obtuvo un número de 27, 24 y 40 plantas $\mathrm{m}^{-2}$, respectivamente (Rojas-García et al., 2016), datos superiores a los encontrados en este estudio, debido posiblemente a las condiciones medio ambientales del lugar como altura $(2240 \mathrm{msnm})$, precipitación media anual $(636,5 \mathrm{~mm})$ y temperatura media anual $\left(15,2^{\circ} \mathrm{C}\right)$, comparadas a este experimento el cual presentó una altura de $2840 \mathrm{msnm}$, precipitación media trimestral de $86,75 \mathrm{~mm}$ y temperatura media trimestral de $12,17^{\circ} \mathrm{C}$.

Durante el periodo de establecimientos se evalúo la variable vigor, la cual se mantuvo en la escala excelente para la mayoría de los tratamientos, aunque se notó un lento establecimiento, principalmente en la mezcla 4, conformada por el pasto azul orchoro (Dactylis glomerata L.), durante el inicio del experimento, comportamiento que coincide con lo reportado por Demanet-Filippi (2014), quien mencionó que el Dactylis glomerata es una especie de lento establecimiento, pero a partir del segundo año productivo se comporta como una planta muy agresiva y competitiva.

Para la edad de 28 días los tréboles dominaron en la mezcla 4, con una baja competitividad del pasto azul orchoro (Dactylis glomerata L), debido quizá al hábito de crecimiento de los tréboles; al respecto Veiga et al. (2009), al comparar mezclas de tréboles anuales con raigrás inglés encontraron resultados similares, en donde los tréboles dominaron la composición de la mezcla en una proporción del 90 \% de la MS total.

Las alturas de los raigrases y el azul orchoro (Dactylis glomerata $\mathrm{L}$ ) en este estudio (Cuadro 4) fueron superiores a las reportadas por Maza-Chamba (2015), quien al evaluar el comportamiento de la mezcla raigrás inglés (Lolium perenne L.), pasto azul (Dactylis glomerata L.) y trébol blanco (Trifolium repens L.), en dos pisos altitudinales, a los 28 días encontró alturas para el raigrás de 24 y $19 \mathrm{~cm}$ y para el pasto azul de 19 y $17 \mathrm{~cm}$, respectivamente. No obstante, el mayor valor obtenido por el Tetrablend 260 fue similar al obtenido por Vargas-Martínez et al. (2018), quienes a los 21 días reportaron alturas de $31,4 \mathrm{~cm}$ para cinco variedades de raigrases perennes. 
Respecto al número de hojas $(\mathrm{H})$, el menor valor obtenido fue para la mezcla 4, con mayor proporción de azul orchoro (Cuadro 4), se asume que a la edad de 28 días esta especie no ha expresado todo su potencial, debido a que su crecimiento es lento en las primeras semanas del ciclo. Sin embargo, una vez este se encuentre establecido, su producción logra igualar o superar la del raigrás. Otros estudios obtuvieron un número de 3,61 hojas por macollo para Dactylis, valor que está por encima al registrado en este estudio, sin embargo, no mencionan la edad de realización de la medida (Beraza-Sánchez et al., 2017; González, 2017).

En el presente estudio el contenido de materia seca fue mayor para la mezcla conformada por azul orchoro (Dactylis glomerata L.), al respecto Rojas-García et al. (2016), encontraron un contenido de MS en el invierno de $3243 \mathrm{~kg} \mathrm{ha}^{-1}$ para la mezcla pasto azul orchoro (Dactylis glomerata L), raigrás y trébol blanco (Trifolium repens L.), en una proporción de 70-20-10, similar a la obtenida en este estudio con la proporción 70-30-10.

Con relación a la incidencia de plagas, se han reportado porcentajes de incidencia del $40 \%$ (moderado según la escala utilizada) para cultivares anuales y en los cultivares perennes porcentajes del $5 \%$ (Mesén \& Sánchez, 2006), similar al encontrado en este estudió para las mezclas con el pasto trojan (Lolium perenne L.). Con respecto a la severidad de enfermedades el mejor comportamiento se evidenció en aquellas mezclas con el pasto Trojan (Lolium perenne L) y tréboles. Según una investigación, los tréboles han desarrollado cierta resistencia a enfermedades, debido a su amplia diversidad genética. En este sentido, se explica la baja presencia de daño causado por enfermedades en la mayoría de las mezclas (Altier et al., 2010).

Para la edad de 35 días la mayoría de las mezclas consiguieron superar 1,8 hojas por planta, destacándose la mezcla 4 con 2, 53 hojas. Teniendo en cuenta esta apreciación, la temperatura promedio de la época y de la zona de evaluación $\left(12,68{ }^{\circ} \mathrm{C}\right)$ influyó en el número de hojas de las mezclas. Condiciones de mayor pluviosidad, son factores que favorecen para la característica de número de hojas en los forrajes (Pérez-Bribiesca et al., 2002).

En un experimento donde se evaluó la asociación de $40 \%$ de ovillo (Dactylis glomerata L.), $20 \%$ de ballico perenne (Lolium perenne L.) y $40 \%$ de trébol blanco (Trifolium repens L.), Rojas-García et al. (2016) obtuvieron una MS de $3099 \mathrm{~kg} \mathrm{ha}^{-1}$ en invierno, valor inferior al reportado en este estudio, debido al porcentaje de asociación de cada especie que para este trabajo fue de 73,52\% de azul orchoro (Dactylis glomerata L.), 17,64 \% de trébol rojo (Trifolium pratense L.) y 8,82\% de trébol blanco (Trifolium repens L.).

El pasto trojan (Lolium perenne) presentó una mayor resistencia a las plagas y patógenos, lo que coincide con Vélez et al. (2002), quienes mencionaron que esta gramínea es poco afectada por plagas y patógenos; de estas últimas la más común es la pudrición de la corona causada por Puccinia coronata, sin embargo, dichos ataques pueden ser controlados con pesticidas.

A la edad de 42 días, el porcentaje de cobertura de trébol fue alto en la mayoría de las mezclas evaluadas con un promedio de $71 \%$, la mezcla 4 fue la que presentó la mayor cobertura de trébol. En un experimento realizado en Alemania, determinó la respuesta de mezclas de diferentes leguminosas y raigrás (Lolium perenne. L.), en dos frecuencias de corte (50 y 30 días), y se encontró que en las épocas con mayor disponibilidad de recurso hídrico las mezclas conformadas por trébol rojo y blanco mostraron mayor vigor y adaptación (Black et al., 2006).

En cuanto al número de hojas $(\mathrm{H})$, la mezcla 4 obtuvo 3,05 hojas, lo que coincide con lo encontrado por Villalobos \& Sánchez (2010) quienes obtuvieron un número de hojas promedio en raigrás de 2,5, lo anterior demuestra que la especie azul orchoro (Dactylis glomerata L) en mezcla presentó una mayor recuperación de reserva de carbohidratos y de la madurez de la hoja para ser pastoreada.

Producciones de materia seca para la mezcla de D. glomerata + leguminosa de $3982 \mathrm{~kg} \mathrm{ha}^{-1}$, para una frecuencia de corte de 45 días en periodo lluvioso fueron obtenidas por Castro-Rincón et al. (2019), lo que difiere de lo encontrado en este estudio para la época de altas precipitaciones donde la mezcla 4 alcanzó una producción de $4708 \mathrm{~kg} \mathrm{ha}^{-1}$ de MS, lo cual puede ser explicado por el mayor nivel de adaptación que posee la especie azul orchoro (Dactylis glomerata L) a la zona de estudio, a su mayor aprovechamiento del nitrógeno suministrado por las gramíneas acompañantes y a una adecuada preparación del terreno y fertilización del mismo. 
En un estudio realizado en Mosquera, Cundinamarca, Colombia se encontró que las gramíneas y leguminosas evaluadas presentaron un vigor de 4,6 y no se presentaron deficiencias nutricionales relacionadas con el estado de vigor de las plantas (Castro-Rincón et al., 2019), lo que coincide con lo encontrado en este estudio, donde la mayoría de tratamientos presentaron un vigor excelente y, por consiguiente, todos los materiales tuvieron una adecuada adaptación a las condiciones medioambientales del sitio de evaluación. En cuanto a la variable plagas y enfermedades estos mismos autores observaron que la incidencia de plagas no fue alta, esto coincide con lo observado en este estudio, en donde la incidencia de plagas fue mínima y la asociación de Lolium sp. con las diferentes especies de tréboles favoreció la resistencia al ataque de plagas y enfermedades.

Para la edad de 28 días, CT fue mayor en la mezcla 3, esto se debió posiblemente a que el trébol rojo (Trifolium pratense L.) es de vigorosa implantación y, se desarrolla muy bien a la sombra, lo que hace que sea agresivo en el establecimiento y de fácil asociación (Cardona-Mejia, 2012).

El L. perenne L. se comportó bien en con pocas precipitaciones y sobre todo en la mezcla 1, debido a que los raigráses son resistentes a la sequía moderada y responden muy bien al riego durante la época seca (Thomas et al., 1999).

El mayor valor para el número de hojas fue de 4,52, obtenido por la mezcla que contenía azul orchoro ( $D$. glomerata L.), al respecto, Ganderats \& Hepp (2003), al evaluar el crecimiento de tres especies (Dactylis glomerata, Lolium perenne L. y F. arundinacea) bajo diferentes condiciones edafoclimáticas, encontraron que D. glomerata es la especie que mejor se adaptó a condiciones de déficit hídrico, debido a que conserva el agua contenida en sus células por más tiempo que otras hierbas, lo que se ve reflejado en una mayor tasa de extensión laminar durante el período estival. La MS fue superior para la mezcla 1. En un experimento similar (Maldonado-Peralta et al., 2017), se reportó un contenido de MS para la época de verano de $4815 \mathrm{~kg} \mathrm{MS} \mathrm{ha}^{-1}$

La incidencia de plagas para la mayoría de los tratamientos fue baja, sin embargo, el tratamiento que no presentó daño alguno por esta variable fue el conformado por la combinación del pasto Trojan + sin riego $+2 \mathrm{t} \mathrm{CaMg}\left(\mathrm{CO}_{3}\right)_{2}$ $\mathrm{ha}^{-1}$. Las semillas procedentes de Nueva Zelanda generalmente traen incorporado el hongo endófito (Neotyphodium occultans), el cual le confiere a las plantas resistencia contra el ataque de insectos plaga y tolerancia al estrés abiótico (Cuesta-Muñoz et al., 2006; Keren, 2017).

Se pudo evidenciar que para la edad de 35 días la mezcla 4 tuvo un buen desarrollo y mantuvo el mayor número de hojas por planta para la época seca, comparado con las demás mezclas, lo que se puede corroborar con lo encontrado por Zhouri et al. (2019), quienes evaluaron la supervivencia a la sequía, la latencia de verano y la productividad de 283 genotipos de Dactylis glomerata, donde el $79 \%$ de la población había sobrevivido después de un verano severo, con una buena perennialidad, alto rendimiento, persistencia y una significativa recuperación. Lo anterior se puede explicar debido a que esta especie cuando se trabaja en mezcla presenta una mayor recuperación de los carbohidratos de reserva, favoreciendo la madurez de la hoja para ser pastoreada.

En Boyacá y Cundinamarca (Colombia), a la edad de 35 días se evaluaron cinco raigrases perennes (tres diploides y dos tetraploides) y tres tréboles (dos rojos y uno blanco), en donde, para la época de bajas precipitaciones la producción de materia seca fue de 1488,4 y 1107,7 $\mathrm{kg} \mathrm{ha}^{-1}$, respectivamente (Castro-Rincón et al., 2019), resultado inferior a lo encontrado en este estudio, debido posiblemente a las condiciones ambientales, del suelo y al asocio de la especie de raigrás Lolium perenne con Trifolium pratense.

En un estudio realizado en Kwazulu - Natal, Sudafrica se encontró en cultivares puros de Lolium perenne L. y en asocio con Trifolium, el vigor del raigrás perenne siguió tendencias de regular a bueno durante el periodo de verano, presentando una mayor invasión de malezas con un potencial de crecimiento, vigor y capacidades de macollamiento (Mckenzie, 1994), lo que coincide con lo encontrado en este estudio para la edad de 35 días, donde la mayoría de los tratamientos presentaron un vigor excelente a excepción de los tratamientos conformados por trojan (Lolium perenne L.) en monocultivo, el cual se mantuvo en una escala de vigor bueno. 
En un experimento realizado en el municipio de Mosquera, Cundinamarca, Colombia a una altura de 2650 msnm, condiciones similares a las de este experimento, se encontró que la cobertura del trébol en las asociaciones alcanzó el 94 \% (Castro-Rincón et al., 2019), equivalente a lo encontrado en este estudio a la edad de 42 días, en donde aquellas asociaciones donde se incluyeron las leguminosas presentaron una cobertura del $100 \%$.

En cuanto a la variable número de hojas, Villalobos \& Sánchez (2010) realizaron una evaluación agronómica del pasto raigrás, donde se determinó 2,68 hojas para la edad de 42 días, lo que coincide con lo encontrado en este estudio, donde la mezcla 4 presentó 3,39 hojas, en este sentido esto se puede deber a la frecuencia de corte, ya que a la edad de 45 días el periodo de recuperación es más largo y, por ende el número de hojas es mayor, obteniendo un forraje menos suculento que a edades de corte más tempranas.

En un experimento realizado en Montecillo, Texcoco, México a una altura de $2240 \mathrm{msnm}$ con $636 \mathrm{~mm}$ de precipitación anual se encontró que la mezcla de raigrás y trébol en verano presentó un rendimiento de MS de 4964 $\mathrm{kg} \mathrm{ha}^{-1}$ (Maldonado-Peralta et al., 2017), lo que difiere a lo reportado en este estudio donde la mezcla 3 tuvo un rendimiento de $3399 \mathrm{~kg} \mathrm{ha}^{-1}$, debido a la baja cantidad de precipitaciones presentadas en esta localidad (86,45 $\left.\mathrm{mm}\right)$.

Para las variables cualitativas se evidenció en la época seca una baja afectación por insectos y agentes fúngicos, al respecto, Cunningham et al. (1993), en un experimento realizado en Australia donde se evaluó la relación del hongo endófito sobre el vigor y efecto de plagas y enfermedades sobre el raigrás perenne, encontraron que la presencia de este mejoró el vigor de las plántulas y aumentó la tolerancia a la sequía de algunos genotipos en ambientes marginales.

El pasto Trebeland 260 mantuvo la mejor altura en época de altas y bajas precipitaciones. En un estudio realizado en el Centro Agropecuario Merengo, en Mosquera, Cundinamarca, Colombia, se encontró que las gramíneas que se evaluaron en asocio con especies leguminosas forrajeras presentaron un promedio de $31,1 \mathrm{~cm}$, lo anterior puede ser explicado por el mayor porcentaje de raigrás tetraploide dentro de la mezcla Trebeland 260, los cuales presentan un establecimiento y desarrollo en la pradera mucho más rápido que las demás especies de gramíneas evaluadas (Castro-Rincón et al., 2019).

La mayor incidencia, severidad de enfermedades y deficiencia nutricional se presentó en la mezcla Trebeland 260 y la mezcla 4, asociado principalmente al ataque de roya (Puccina sp.), el cual fue visible de manera leve en los cuatro cortes, al respecto Estrada-Álvarez (2002), afirmó que el principal problema que se presenta con algunos tetraploides en las condiciones del trópico es su adaptación, pues mientras algunos son muy susceptibles a enfermedades como la roya, otros desaparecen muy rápidamente de la pradera por las condiciones climáticas o de suelos, haciendo necesarias costosas resiembras.

\section{Conclusiones}

A través de la asociación (gramínea y leguminosa) se generó un mayor rendimiento de MS, cobertura, altura y número de hojas, en comparación con el monocultivo de gramíneas puras, alargando el periodo de producción o vida útil de las especies de ryegrass, mayor adaptación a factores de variabilidad climática y deficiencias nutricionales.

La producción de MS entre épocas y frecuencias de corte, se incrementó para las mezclas de gramíneas y leguminosas. La mejor mezcla fue la compuesta por D. glomerata, Trifolium repens L. y Trifolium pratense L.

Se determinó que las asociaciones de gramíneas y leguminosas se mantuvieron en una escala sin daño y con daño leve para las variables incidencia de plagas y enfermedades, severidad de enfermedades y deficiencias nutricionales, con ventajas comparativas frente a los monocultivos de gramíneas puras.

Durante la época de altas y bajas precipitaciones en las diferentes edades evaluadas no se observaron efectos asociados a la interacción de los factores riego y enmienda. 


\section{Agradecimientos}

Los autores agradecen en especial a la Corporación Colombiana de Investigación Agropecuaria (AGROSAVIA) y al macroproyecto denominado "Mejoramiento de la oferta forrajera, optimización de sistemas de alimentación y aseguramiento de la calidad e inocuidad de la leche en el trópico alto del departamento de Nariño", ejecutado mediante recursos del Sistema General de Regalías.

\section{Referencias}

Altier, N., Rebuffo, M., \& Cabrera, K. (Eds.). (2010). Enfermedades y plagas en pasturas. Instituto Nacional de Investigación Agropecuaria. http://www.ainfo.inia.uy/digital/bitstream/item/7729/1/st-183-2010-p.19-35.pdf

Beraza-Sánchez, S., Guerisoli-Pereira, G., \& Rodríguez-Echenique, E. (2017). Morfogenésis en Festuca arundinacea y Dactylis glomerata [Tesis de pregrado, Universidad de la República de Montevideo]. Repositorio de la Universidad de la República de Montevideo. https://www.colibri.udelar.edu.uy/jspui/bitstream/20.500.12008/18614/1/TTS_ BerazaS\%c3\%a1nchezSantiago.pdf

Black, A. D., Moot, D. J., \& Lucas, R. J. (2006). Spring and autumn establishment of Caucasian and white clovers with different sowing rates of perennial ryegrass. Grass and Forage Science, 61(4), 430-441. https://doi.org/10.1111/j.13652494.2006.00552.x

Cadena-Guerrero, M. M., García-Dávila, M. A., \& Castro, E. (2019a). Phenotypic stability of genotypes Lolium sp. In the high tropic of Nariño, Colombia. Agronomía Mesoamericana, 30(2), 483-495. https://doi.org/10.15517/am.v30i2.34307

Cadena-Guerrero, M. M., García-Dávila, M. A., Meneses-Buitrago, D. H., Morales-Montero, S. P., \& Castro-Rincón, E. (2019b). Adaptation of ten cultivars of Lolium sp. In the high tropic of Nariño, Colombia. Agronomía Mesoamericana, 30(1), 165-178. https://doi.org/10.15517/am.v30i1.34094

Camacho-García, J. L., \& García-Muñiz, J. G. (2003). Dry matter yield and nutritive value of four alfalfa varieties associated with white clover, perennial ryegrass, tall fescue and orchard grass. Veterinaria Mexico, 34(2), 149-177. https://www. redalyc.org/articulo.oa?id $=42334204$

Cardona-Mejia. (2012). Pastos y especies forrajeras [Modulo]. Federación Colombiana de Ganaderos. https://www.fedegan. org.co/modulo-pastos-y-especies-forrajeras

Carulla, J. E., Cárdenas, E., Sánchez, N., \& Riveros, C. (2003, 1-2 setiembre). Valor nutricional de los forrajes más usados en los sistemas de producción lechera especializada de la zona andina colombiana. En Eventos y Asesorías Agropecuarias EU (Ed.), Seminario Nacional de Lechería Especializada: Bases Nutricionales y su Impacto en la Productividad, Medellín, Colombia (pp.1-16). Eventos y Asesorías Agropecuarias EU.

Castro-Rincon, E., Carulla-Fornaguera, J. E., \& Cárdenas-Rocha, E. A. (2019). Adaptación de gramíneas asociadas con Lotus uliginosus Schkuhr en el trópico alto de Colombia. Pastos y Forrajes, 42(1), 13-22. http://scielo.sld.cu/pdf/pyf/ v42n1/2078-8452-pyf-42-01-13.pdf

Cobo, J. A. (2003). El suelo y el agua en la producción de pastos. Cobo Lemos, José Alirio.

Cuesta-Muñoz, P. A., Bolaños-Alomia, A., \& Betancourth, C. A. (2006, diciembre 15). Nuevas especies forrajeras para mejorar la competitividad de los sistemas de produccion de leche del Altiplano de Nariño [Día de campo]. Corporación Colombiana de Investigación Agropecuaria, Pasto, Colombia. https://repository.agrosavia.co/ handle/20.500.12324/18646

Agron. Mesoam. 32(2):538-555, mayo-agosto, 2021 
Cunningham, P. J., Foot, J. Z., \& Reed, K. F. M. (1993). Perennial ryegrass (Lolium perenne) endophyte (Acremonium lolii) relationships: the Australian experience. Agriculture, Ecosystems and Environment, 44(1-4), 157-168. https://doi. org/10.1016/0167-8809(93)90044-P

de Mendiburu, F. (2017, 12 de septiembre). Package 'agricolae’. The Comprehensive R Archive Network. https://cran.r-project. org/web/packages/agricolae/agricolae.pdf

Demanet-Filippi, R. (2014). Manual de Especies Forrajeras [Manual]. Consorcio Lechero. http://www.consorciolechero.cl/ industria-lactea/wp-content/uploads/2014/10/Manual-de-Especies-Forrajeras-2014-Watts.pdf

Edmeades, D. C., Shannon, P. W., Pringle, R. M., \& Mansell, G. P. (2012). Effects of lime on pasture production on soils in the north island of new zealand: 4. Predicting lime responses. New Zealand Journal of Agricultural Research, 27(3), 371-382. https://doi.org/10.1080/00288233.1984.10430638

Estrada-Álvarez, J. (2002). Pastos y forrajes para el trópico colombiano. Editorial Universidad de Caldas.

ETHzürich. (2016, 19 de junio). Identification of novel rice blast resistance sources. Institute of Molecular Plant Biology. https:// impb.ethz.ch/research/reseach-pb/research-pb/research-nkb/resistancessources.html

Ganderats, S., \& Hepp, C. (2003). Mecanismos de crecimiento de Lolium perenne, Festuca arundinacea y Dactylis glomerata en la zona intermedia de Aysen. Agricultura Tecnica, 63(3). 259-265. https://doi.org/http://dx.doi.org/10.4067/S036528072003000300005

González, K. (2017, 25 de julio). Pasto azul (Dactylis glomerata).Zootecnia y Veterinaria es mi pasión. https://zoovetesmipasion. com/pastos-y-forrajes/pasto-azul-dactylis-glomerata/

Keren, Z. (2017). Growth and feed quality of five perennial ryegrass (Lolium perenne L.) cultivars under three water treatments [Magister Science Thesis, Lincoln University]. Lincoln University Repository. https://researcharchive.lincoln.ac.nz/ bitstream/handle/10182/8825/Zhong_MApplSc.pdf?sequence=3

Maldonado-Peralta, M. de los ángeles, Rojas-Garcia, A. R., Torres-Salado, N., Herrera-Pérez, J., Cancino, S. J., Ríos, J. V., Hernández-Garay, A., \& Hernández-Guzmán, F. J. (2017). Productivity of orchard grass (Dactylis glomerata L.) alone and associated with perennial ryegrass (Lolium perenne L.) and white clover (Trifolium repens L.). Revista Brasileira de Zootecnia, 46(12), 890-895. https://doi.org/10.1590/S1806-92902017001200003

Maza-Chamba, W.(2015). Evaluación de tres especies forrajeras: rye grass inglés (Lolium perenne L.), pasto azul (Dactylis glomerata L.) y trebol blanco (Trifolium repens L.) en dos pisos altitudinales del cantón Loja [Tesis de pregrado, Universidad Nacional de Loja]. Repositorio de la Universidad Nacional de Loja. https://dspace.unl.edu.ec/jspui/ bitstream/123456789/11584/1/TESIS\%20WILMER\%20ALCIDES\%20MAZA\%20CHAMBA.pdf

McCarthy, S., Wims, C., Lee, J., \& Donaghy, D. (2016). Perennial ryegrass grazing management in spring [Brochure]. Dairy NZ. https://www.dairynz.co.nz/media/2634153/perennial-ryegrass-grazing-guide-web.pdf

Mckenzie, F. R. (1994). Managing Lolium perenne L. (perennial ryegrass) in a sub-tropical environment in KwazuluNatal, South Africa [Postdoctoral Thesis, University of Natal Pietermaritzburg]. University Kwazulu - Natal Repository. https://researchspace.ukzn.ac.za/xmlui/bitstream/handle/10413/10910/McKenzie_Frank_R_1994. pdf? sequence $=1 \&$ isAllowed $=\mathrm{y}$

Mesén, M., \& Sánchez, W. (2006). Evaluación de gramíneas forrajeras en la zona alta lechera de Costa Rica. Alcances Tecnológicos, 4(1), 29-36. https://doi.org/10.35486/at.v4i1.112 ISSN 2215-3608 doi:10.15517/am.v32i2.41265 
Morales, A., León, J., Cárdenas, E., Afanador, G., \& Carulla, J. (2013). Calidad de leche, digestibilidad in vitro de la materia seca y producción en vacas alimentadas con grampíneas solas o asociadas con Lotus uliginosus. Revista de la Facultada de Medicina Veterinaria y de Zootecnia, 60(I), 32-48. http://www.scielo.org.co/pdf/rfmvz/v60n1/v60n1a04.pdf

Pérez-Bribiesca, T., Hernández-Garay, A., Pérez-Pérez, J., Herrera-Haro, J., \& Bárcena-Gama, R. (2002). Respuesta productiva y dinámica de rebrote del ballico perenne a diferentes alturas de corte. Técnica Pecuaria en México, 40(3), 251-263. https://doi.org/10.22319/rmcp.v40i3.1297

R Development Core Team 3.0.1. (2013). A language and environment for statistical computing. R Foundation for Statistical Computing. http://www.r-project.org

Reynolds, C. (2000). Forage evaluation using measurements of energy metabolism. In. D. Givens, R. Axford, \& E. Owen (Eds.). Forage evaluation ruminant nutrition (pp. 95-113). CAB International Publishing.

Rojas- García, A. R., Hernández-Garay, A., Ayala, W.,Mendoza-Pedroza, S. I. M., Cancino, S. J., Vaquera-Huerata, H. V., \& Santiago-Ortega, M. A. S. (2016). Comportamiento productivo de praderas con distintas combinaciones de ovillo (Dactylis glomerata L.), ballico perenne (Lolium perenne L.) y trébol blanco (Trifolium repens L.). Revista de La Facultad de Ciencias Agrarias, 48(2), 57-68. https://www.redalyc.org/pdf/3828/382847506012.pdf

Sánchez,L., \& Villaneda, E. (2009). Renovación y Manejo De Praderas En El Trópico Alto Colombiano [Folleto]. Repositorio de la Corporación Colombiana de Investigación Agropecuaria. https://repository.agrosavia.co/handle/20.500.12324/12867

Thomas, H., James, A. R., \& Humphreys, M. W. (1999). Effects of water stress on leaf growth in tall fescue, Italian ryegrass and their hybrid: Rheological properties of expansion zones of leaves, measured on growing and killed tissue. Journal of Experimental Botany, 50(331), 221-231. https://doi.org/10.1093/jxb/50.331.221

Toledo, J. M. (Ed.). (1982). Manual para la evaluación agronómica: Red Internacional de Evaluación de Pastos Tropicales. Centro Internacional de Agricultura Tropical. http://ciat-library.ciat.cgiar.org/Forrajes_Tropicales/pdf/Books/split/ Manual_Evaluacion.pdf

Vargas-Martínez, J. D. J., Sierra-Alarcón, A., Benavidez-Cruz, J., Avellaneda-Avellaneda, Y., \& Ariza-Nieto, C. (2018). Establecimiento y producción de raigrás y tréboles en dos regiones del trópico alto colombiano. Agronomía Mesoamericana, 29(1), 177-191. https://doi.org/10.15517/ma.v29i1.28077

Veiga, M., Botana, A., Resch, C., Pereira-Crespo, S., Dagnac, T., Valladares, J., Díaz, N., Fernández-Lorenzo, B., \& FloresCalvete, G. (2009, 26-29 abril). Comparación de una mezcla de tréboles anuales con raigrás inglés en un sistema de pastoreo con vacas de leche. Innovación Sostenible en Pastos: hacia una Agricultura de Respuesta al Cambio Climático, Coruña, España. Pastos, 45(2), 235-240. http://ciam.gal/uploads/publicacions/1238archivo.pdf

Vélez, M., Hincapie, J., Matamoros, I., \& Santillan, R. (2002). Producción de Ganado Lechero en el Trópico (4ª Ed.). Zamorano Academic Press.

Villalobos, L., \& Sánchez, J. M. (2010). Evaluación agronomica nutricional del pasto ryegrass perenne tetraploide (Lolium perenne) producido en lecherias de las zonas altas de costa rica. ii. valor nutricional. (Spanish). Agronomía Costarricense, 34(1), 31-42. http://doi.org/10.15517/RAC.V34I1.6698

Wickham, H. (2016). ggplot2: Elegant Graphics for Data Analysis. Springer-Verlag New York. https://ggplot2.tidyverse.org

Zhouri, L., Kallida, R., Shaimi, N., Barre, P., Volaire, F., Gaboun, F., \& Fakiri, M. (2019). Evaluation of cocksfoot (Dactylis glomerata L.) population for drought survival and behavior. Saudi Journal of Biological Sciences, 26(1), 49-56. https://doi.org/10.1016/j.sjbs.2016.12.002 